\title{
Dual photometric-conductometric detector for microfluidic chip
}

\author{
Feng Shen, Yong Yu*, Qi Kang \\ Key Laboratory of Microgravity, Institute of Mechanics, Chinese Academy of Sciences, Beijing \\ 100190, China
}

\begin{abstract}
A dual simultaneous photometric-conductometric detector for microfluidic chip is reported. Two different detect methods confocal LIF and moveable $\mathrm{C}^{4} \mathrm{D}$ were combined together. They shared a common detection cell and could respond simultaneously. The dual detector offered possibility of measurement one analyte in two different ways and was advantageous in analyses of mixtures containing organic and inorganic ions. To improve the separation efficiency, pinch injection was carried out by a series of electrokinetic manipulations. Rhodamine B was used to evaluate the performance of the dual detector. The dual detector had highly sensitivity and could offer simultaneous information.
\end{abstract}

Keywords: Microfluidic, lab-on-a-chip, $\mu$-TAS, laser-induced fluorescence (LIF), capacitively coupled contactless conductivity detection $\left(\mathrm{C}^{4} \mathrm{D}\right)$

\section{INTRODUCTION}

Various types of microfluidic devices and detection techniques have been used in micro-total analysis systems ( $\mu$-TAS) or lab-on-a-chip[1], with the aim of developing miniature-sized bio/chemical systems, which have numerous potential benefits including low regent consumption, high sensitivity, automation of processes, and short analysis time[2]. As one of the most important parts in microchip, many detection methods have been investigated, such as fluorescence detection, electrochemistry and mass spectrometry [3]. LIF is the most widespread detection method because of its high sensitivity (especially the confocal LIF mode) and ease of coupling with a microchip [4]. Besides, $\mathrm{C}^{4} \mathrm{D}$ is receiving increased attention, because it is a versatile and sensitive method and can be easily miniaturized on the microchip. This method provides a means to measure some ions that are not easily detected by fluorescence. The moveable $C^{4} \mathrm{D}$ could detect the single at different points along the separation channel and could offer distinct improvements [5].

As the field of microfluidic devices continuous its rapid developments, there are urgent needs for developing combined detection systems. Due to the diversity of components in real samples, it is usually difficult to determinate all components by using a single detection method. A variety of dual detector schemes have thus been proposed for capillary electrophoresis (CE) [6,7], but rarely in connection with microfluidic chip[8-11]. Wang and Pumera [8] described a dual conductivity/amperometric microchip detection system to offer simultaneous detection of both electroactive and ionic species. Ewing's group [9] demonstrated a simultaneous LIF and amperometric detection in microchip CE. Wang's group [10] demonstrated a simultaneous electrochemical and electrochemiluminescence detection scheme for both microchip and conventional CE systems. Using a blue light-emitting diode as excitation source, Chen et al.[11] recently reported a compact dual fluorescence and conductivity detector which sensitivity was not high. Up to now, none has combined the highly sensitive confocal LIF and moveable $\mathrm{C}^{4} \mathrm{D}$ together for microfluidic chip.

In this paper, we report a novel dual detector with simultaneous confocal LIF and moveable $C^{4} \mathrm{D}$ at the same place of the microfluidic chip. The LIF was designed according to the confocal principle and the $C^{4} \mathrm{D}$ was performed using a moveable electrode plate. The coupling of the two detection methods offered possibility of measurement one analyte in two different ways for making obtained results more reliable. Moreover, it facilitated the measurement of analytes with different physical properties, so it could enhance the detection characterization of sample and could offer simultaneous detection information of both ionic and fluorescent compounds. The detection conditions were optimized and the injection conditions within the microchannel were studied. The performance was demonstrated by simultaneous detection of Rhodamine B. The limit of detection (LOD) were less than $5 \mathrm{nM}(\mathrm{S} / \mathrm{N}=8)$ and $0.1 \mu \mathrm{M}(\mathrm{S} / \mathrm{N}=6)$ of confocal LIF and $C^{4} \mathrm{D}$ respectively.

\footnotetext{
*yuyong@imech.ac.cn
}

ICEM 2008: International Conference on Experimental Mechanics 2008, edited by Xiaoyuan He, Huimin Xie, Yilan Kang, Proc. of SPIE Vol. 7375, 73751P - () 2009 SPIE · CCC code: 0277-786X/09/\$18 · doi: 10.1117/12.839069 


\section{EXPERIMENTAL}

\subsection{Reagents}

The Borax run buffer solution (10 mM, pH 9.0) was prepared. High-purity deionized water (18.2 M $\Omega$ ) was purified by a Millipore Simplicity for preparation of all aqueous solutions. Rhodamine B was obtained from Sigma-Aldrich Co. (St. Louis, MO). Borax was purchased from Beijing Reagents Co. (Beijing, China). Stock solution of rhodamine B was prepared by dissolving it in the run buffer. All solutions were filtered using $0.2 \mu \mathrm{m}$ syringe filter before use. All reagents used were analytical grade.

\subsection{Experimental setup}

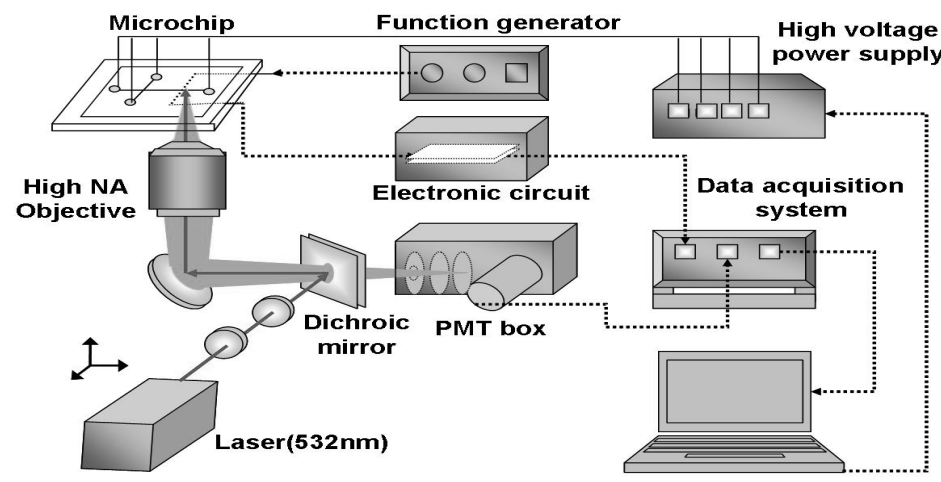

Fig. 1. Experimental setup for the dual confocal $\mathrm{LIF} / \mathrm{C}^{4} \mathrm{D}$ detector.

The homemade dual confocal LIF/C $\mathrm{C}^{4} \mathrm{D}$ detector consisted of a photometric detection system, a conductometric detection system and the high voltage supplier and date acquisition system, displayed in details in Fig. 1. For the photometric detection system, a 532-nm semiconductor laser (output power $50 \mathrm{~mW}$ ) was used as light source. The laser power was adjusted by a circular variable attenuator. The laser beam was passed through a 532-nm band-pass filter (Shenyang HB Optical Technology Co., Shenyang, China), reflected by two mirrors (one was dichroic mirror) and focused by a high NA objective (0.65 NA, 2.95-mm-long working distance, Chongqing MIC Optical \& Electrical Instrument Co.) to form a small spot (about $0.1 \mathrm{~mm}$ ) in the separation channel. Alignment of the microchip position was adjusted by an X-Y-Z translation stage (Beijing Optical Instrument Factory, Beijing, China). The excited fluorescence was collected by the same objective, then passed though the same dichroic mirror (DM575, Shenyang HB Optical Technology Co.), a spatial filter (diameter $500 \mu \mathrm{m}$ ) and two pieces of $580 \mathrm{~nm}$ long-pass filters (Shenyang HB Optical Technology Co.) before being detected by a photomultiplier tube (PMT CR114, Hamamatsu, Japan).

For the conductometric detection system, the $\mathrm{C}^{4} \mathrm{D}$ was performed using a moveable electrode plate containing two electrodes. An Agilent 33120A function generator (Agilent, Palo Alto, CA) was used for generating a sinusoidal signal (usually with a frequency of $289 \mathrm{kHz}$ and with peak-to-peak amplitude of $5 \mathrm{Vp}-\mathrm{p}$ ) applied to one of the electrodes. The electronic circuitry was connected to another electrode. The circuitry was designed according to the reported scheme [12] and completed by adding a low-pass filter followed by a changeable gain amplifier. There were three options for amplificatory multiple of inductive current: $300 \mathrm{X}, 600 \mathrm{X}$ and $1500 \mathrm{X}$. The electronic circuitry was placed in a shielding box to protect the electronics from external electric fields.

The high voltage supplier with an adjustable voltage range between 0 and $+5000 \mathrm{~V}$ was used for sample injection and separation. A HP 34970A data acquisition system (Agilent, Palo Alto, CA) was used for collecting both fluorescence and conductometric signals simultaneously. Data treatment was performed using HP BenchLink DataLogger program.

\subsection{Design of the detection cell}

A cross-pattern PMMA microfluidic chip with $50 \mathrm{~mm}$ of separation channel and $8 \mathrm{~mm}$ of injection channel was used, shown in Fig. 2. The dimensions of the channel are $25 \mu \mathrm{m}$ deep, $125 \mu \mathrm{m}$ wide at the top, and $75 \mu \mathrm{m}$ wide at the bottom. One side of the channel was covered by a $50 \mu \mathrm{m}$-thin PMMA membrane. 
The dual detector shared a common detection cell, shown in details in Fig. 2. The electrodes on the moveable plate were two rectangular-shaped, $10 \mu \mathrm{m}$-thick copper films $(0.8 \mathrm{~mm} \times 28.2 \mathrm{~mm})$, which were fabricated on a PMMA plate by vacuum vapor deposition method, with a distance of $800 \mu \mathrm{m}$ between them. The electrodes were placed in an antiparallel orientation and the end side of the electrodes was widened to $4 \mathrm{~mm}$ to facilitate the electrical connection. Two copper wires were fixed at the end side of the electrodes using silver paint and then a quick-setting epoxy. The electrode plate could move along the separation channel, and detection in different position of the channel would also be available.

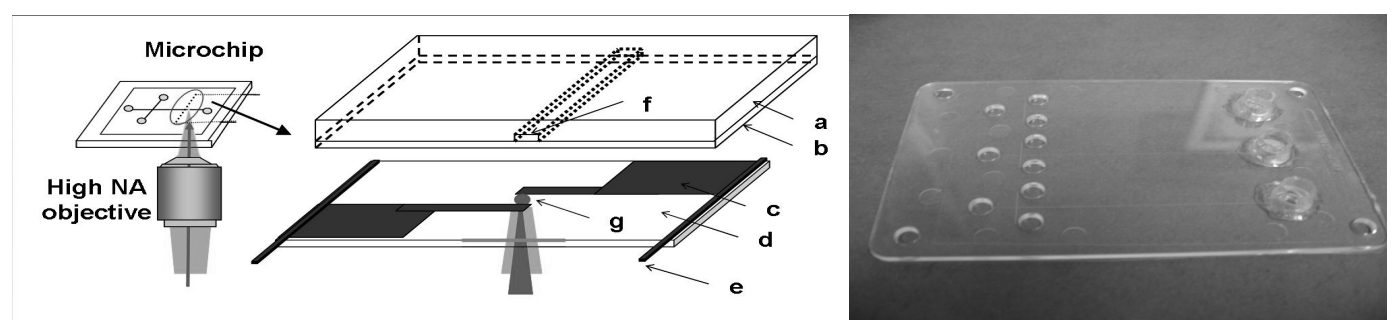

Fig. 2. Schematic diagram of the detection cell and microfluidic chip. (a)microfluidic chip; (b)chip cover(50 $\mu \mathrm{m})$; (c)copper electrodes $(10 \mu \mathrm{m})$; (d)electrode plate; (e) copper wires; (f) channel; (g)laser focus.

\subsection{Procedure}

The channels of the PMMA chip were sequentially flushed with $0.1 \mathrm{M} \mathrm{NaOH}$, deionized water, and $10 \mathrm{mM}$ borate buffer before use. Sample reservoir was filled with the sample solution and others with run buffer solution. The chip was placed on a homemade Polyvinyl Chloride platform fixed on the X-Y-Z stage. The chip position was adjusted to make the focus point of the laser in middle of the two electrodes. Then the chip was operated under sample injection and separation process by a series of electrokinetic manipulations.

\section{RESULTS}

\subsection{Electrokinetic injection techniques}

The separation efficiency of the microfluidic chip is influenced to a significant degree by the flow field conditions within the injection microchannel. Handing of the fluid is generally carried out by a series of electrokinetic manipulations. An understanding of the mechanisms governing electrokinetic manipulations is beneficial in the design and operation of microfluidic devices. With the pinched injection, the volume of the sample plug can be accurately controlled and is time independent, enabling a constant volume to be injected [13]. For the pinched injection, potentials were applied to the analyte, the buffer, and the waste reservoirs with the analyte waste reservoir grounded in order to control the injection plug shape. In the separation step, the potential was applied to the buffer reservoir with the waste reservoir grounded and with analyte and analyte waste reservoirs at appropriate potential.
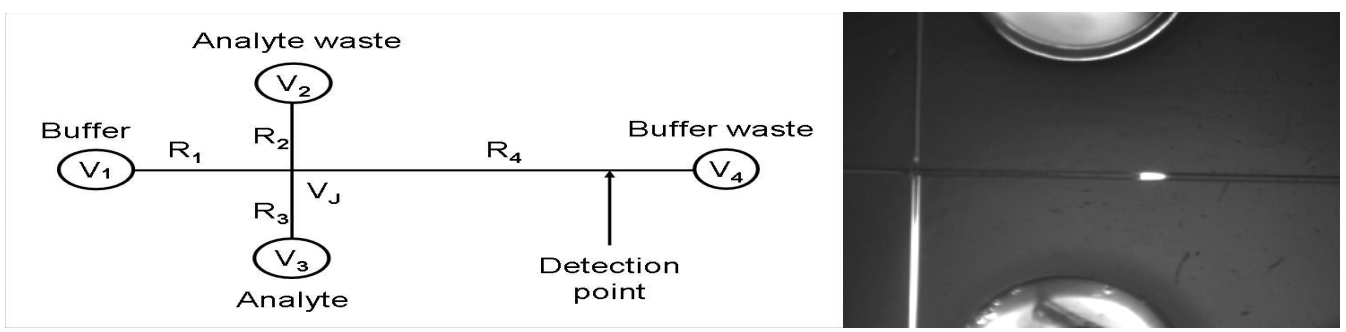

Fig. 3. Schematic of microfluidic chip and image of a separation of fluorescein.

The mathematical model for the injection and separation steps can be written as

Injection step

$$
V_{3}=V_{\max }, V_{2}=0, V_{1}=V_{4}=V_{J}, V_{J}=\frac{\frac{V_{1}}{R_{1}}+\frac{V_{3}}{R_{3}}+\frac{V_{4}}{R_{4}}}{\frac{1}{R_{1}}+\frac{1}{R_{2}}+\frac{1}{R_{3}}+\frac{1}{R_{4}}}
$$


Separation step

$$
V_{1}=V_{\max }, V_{4}=0, V_{2}=V_{3}=V_{J}^{\prime}, \quad V_{J}^{\prime}=\frac{\frac{V_{1}}{R_{1}}+\frac{V_{2}}{R_{2}}+\frac{V_{3}}{R_{3}}}{\frac{1}{R_{1}}+\frac{1}{R_{2}}+\frac{1}{R_{3}}+\frac{1}{R_{4}}}
$$

Equations 1and 2 can quickly calculate each electric potential required in the injection and separation steps.

Table. 1. Potential in the experiment

\begin{tabular}{|lccccc|}
\hline \multicolumn{5}{c|}{ Electric potential strengths $(\mathrm{V})$} \\
Injection & Buffer $\left(\mathrm{V}_{1}\right)$ & Analyte waste $\left(\mathrm{V}_{2}\right)$ & Analyte $\left(\mathrm{V}_{3}\right)$ & Buffer waste $\left(\mathrm{V}_{4}\right)$ & Time $(\mathrm{s})$ \\
Separation & 500 & 0 & 1000 & 600 & 20 \\
& 1600 & 1400 & 1400 & 0 & 180 \\
\hline
\end{tabular}

After the analyte has been pumped electrophoretically and electroosmotically from the analyte reservoir to the analyte waste reservoir with the buffer from the buffer reservoir and the waste reservoir traveling toward the analyte waste reservoir, the voltages are manually switched from the injection step to the separation step. In Fig. 3 the image shows a separation of fluorescein within the separation channel. The injection plug length depends largely on both the flow pattern of the analyte and the dimensions of the injection cross. The volume of the injection plug is about $100 \mathrm{pL}$ with pinched injection.

\subsection{Photometric part of the dual detector}

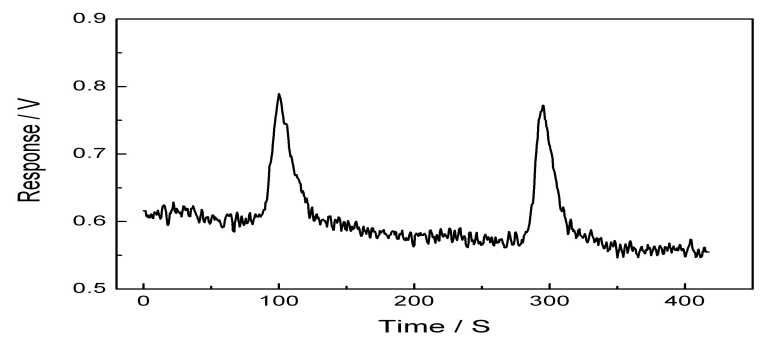

Fig. 4. Electropherogram of $5 \mathrm{nM}$ Rhodamine B for two times with LIF detection. run buffer, borax (10 mM, $\mathrm{pH} 9.0)$; time RSD, $2.3 \%$, peak value RSD, $7.4 \%, n=10$.

The sensitivity of LIF depends on various parameters [4]. To develop our high-sensitivity LIF detection system, a highefficiency PMT and a relatively high NA objective with lower magnification for high collection efficiency of emitted photons were used, and excitation laser power was optimized so as not to overly saturate photon emission from fluorophores. A linear range of two orders of magnitude (from $8 \mathrm{nM}$ to $0.8 \mu \mathrm{M}$; correlation coefficients, >0.998) was obtained for Rhodamine B, and the limit of detection (LOD) was less than $5 \mathrm{nM}(\mathrm{S} / \mathrm{N}=8)$ (as shown in Fig. 4), which sensitivity was lower than that of photo counting model detection $(8.5 \mathrm{pM})$ [14] and much higher than absorbance detection $(0.95 \mu \mathrm{M})$ [15], LIF optical fiber detection $(0.1 \mu \mathrm{M})[16]$ and photothernal technique detection $(2 \mu \mathrm{M})[17]$ for Rhodamine B. The results demonstrated high reproducibility and sensitivity.

\subsection{Conductometric part of the dual detector}

Response of the $\mathrm{C}^{4} \mathrm{D}$ was strongly dependent upon the frequency and amplitude of the applied voltage. The optimum working frequency must be found experimentally for the given detector design and set of conditions. The influences of the applied ac voltage frequency on peak area and signal-to-noise rate (SNR) were shown in Fig. 5. The curves were plotted over the $0-500 \mathrm{kHz}$ range for Rhodamine B. The peak area increased slowly between 60 and $190 \mathrm{kHz}$, then more rapidly (reaching the maximum at $289 \mathrm{kHz}$ ), and decreased sharply thereafter. When the frequency was lower than 60 $\mathrm{kHz}$, there was no response for any running buffer or sample. The SNR had the same tendency as the peak area (except at $240 \mathrm{kHz}$ ). High frequency or high voltage would induce unstable response and baseline drift, which could lead to the decrease of SNR. 
The effect of the applied ac voltage amplitude on the peak area and SNR were illustrated in Fig. 6. The peak area increased with the applied ac voltage (Vp-p) from 2 to $8 \mathrm{Vp}-\mathrm{p}$. When the voltage was lower than $1 \mathrm{Vp}-\mathrm{p}$, there was no response. The SNR steadily increased between $1 \mathrm{Vp}-\mathrm{p}$ and $5 \mathrm{Vp}$-p, and decreased after $5 \mathrm{Vp}-\mathrm{p}$. The most favorable SNR characteristics were obtained at the frequency of $289 \mathrm{kHz}$ and the voltage of $5 \mathrm{Vp}-\mathrm{p}$.

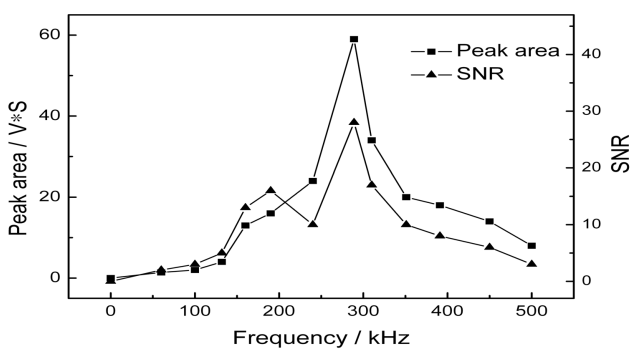

Fig. 5. Influence of the frequency upon the peak area and SNR of $80 \mu \mathrm{M}$ Rhodamine B with $\mathrm{C}^{4} \mathrm{D}$. Vpp, $5 \mathrm{~V}$; amplificatory multiple, $600 \mathrm{X}$.

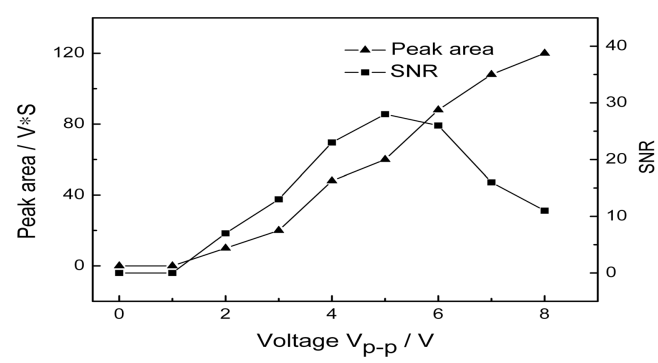

Fig. 6. Influence of the voltage amplitude upon the peak area and SNR with $\mathrm{C}^{4} \mathrm{D}$. frequency, 289 $\mathrm{kHz}$.

The intensity of the response and the relative peak value would be remarkably increased with increasing the amplificatory multiple. The amplificatory multiple of $600 \mathrm{X}$ was chosen because the most favorable response characteristics could be achieved at this amplificatory multiple. A linear range of nearly two orders of magnitude (from $0.25 \mu \mathrm{M}$ to $20 \mu \mathrm{M}$; correlation coefficients, >0.997) was obtained for Rhodamine $\mathrm{B}$, and the LOD was $0.1 \mu \mathrm{M}(\mathrm{S} / \mathrm{N}=6)$, which sensitivity was higher than that of inorganic ions detection (about $1 \mu \mathrm{M}$ ) reported using $\mathrm{C}^{4} \mathrm{D}[8,11]$.

\subsection{Simultaneous detection}

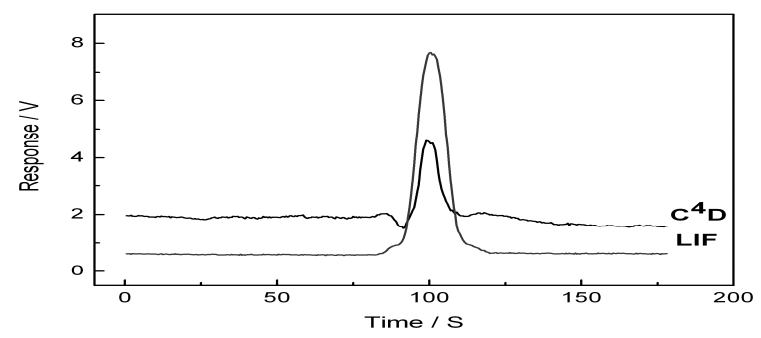

Fig. 7. Electropherogram of $2 \mu \mathrm{M}$ Rhodamine B with simultaneous dual LIF/C $\mathrm{C}^{4} \mathrm{D}$ detector.

The electropherogram for the simultaneous confocal LIF and $\mathrm{C}^{4} \mathrm{D}$ detection of Rhodamine B was shown in Fig. 7. The simultaneous responses were obtained at the same time and the time RSD was $5.3 \%(n=9)$. The sensitivity of LIF was higher than that of the $\mathrm{C}^{4} \mathrm{D}$ which was more sensitive to ions detection. The dual detector offered possibility of measurement one analyte in two different ways for making obtained results more reliable.

\section{CONCLUSIONS}

We have demonstrated a novel simultaneous dual confocal LIF/C ${ }^{4} \mathrm{D}$ detector for microfluidic chip. The dual detector combines the respective response advantages of LIF and $\mathrm{C}^{4} \mathrm{D}$ methods to different analytes and facilitates the measurement of analytes with different physical properties, thus it enhanced the detection characterization of sample and could offer simultaneous response to ionic and fluorescent compounds. Moreover, the dual detector offered the possibility of simultaneous measurement on of one analyte in two different ways and would be very useful in daily clinical analysis for making obtained results more reliable. Additionally, the two detection methods were mutually independent and provided opportunities to optimize performance for both detection and separation. 


\section{ACKNOWLEDGMENT S}

The authors gratefully acknowledge the financial support provided to this study by the Innovation Fund of Chinese Academy of Sciences under Grant Nos. 0830082XM1.

\section{REFERENCES}

[1] A. Manz, N. Graber and H. M. Widmer, Sensor. Actuators B, 7, 244-248 (1990).

[2] G. M. Whitesides, Nature, 442, 368-373 (2006).

[3] K. Uchiyama, H. Nakajima and T. Hobo, Anal. Bioanal. Chem., 379, 375-382 (2004).

[4] B. G. Jung, Y. G. Zhu, and J. G. Santiago, Anal. Chem., 79, 345-349 (2007).

[5] J. Wang, G. Chen, and A. Muck Jr., Anal. Chem., 75, 4475-4479 (2003).

[6] M. Novotny, F. Opekar, I. Jelínek, and K. Stulík, Anal. Chim. Acta, 525, 17-21 (2004).

[7] F. Tan, B. C. Yang, and Y. F. Guan, Anal. Sci., 21, 583-585 (2005).

[8] J. Wang and M. Pumera, Anal. Chem., 74, 5919-5923 (2002).

[9] J. A. Lapos, D. P. Manica, and A. G. Ewing, Anal. Chem., 74, 3348-3353 (2002).

[10] H. B. Qiu, E. K. Wang, et al., Electrophoresis, 26, 687-693 (2005).

[11] C. Liu, Y. Y. Mo, Z. G. Chen, X. Li, O. L. Li, and X. Zhou, Anal. Chim. Acta, 621, 171-177 (2008).

[12] J. A. Fracassi da Silva, and C. L. do Lago, J. Chromatogr., A, 942, 249-258 (2002).

[13] S. C. Jacobson, R. Hergenroder, et al., Anal. Chem., 1994, 66, 1107-1113 (1994).

${ }^{[14]}$ J. C. Fister, S. C. Jacobson, L. M. Davis, and J. M. Ramsey, Anal. Chem., 70, 431-437 (1998).

${ }^{[15]}$ G. E. Collins, Q. Lu, N. Pereira, and P. Wu, Talanta, 72, 301-304 (2007).

[16] C. H. Lin, G. B. Lee, S. H. Chen, and G. L. Chang, Sensor. Actuators A, 107, 125-131 (2003).

[17] K. Katayama, K. Kikutani, and T. Kitamori, Anal. Sci., 23, 639-643 (2007). 